\title{
Use of lipid bilayers as support for biomolecules integration in OTFT biosensors
}

\author{
S. Cotrone ${ }^{a}$, M. Magliulo ${ }^{a}$, M.D. Angione ${ }^{a}$, A. Mallardi ${ }^{b}$, M. Ambrico ${ }^{c}$; T. Ligonzo ${ }^{d}$, M. Colella ${ }^{e}$, \\ G. Palazzo ${ }^{\text {, }}$ L. Torsi ${ }^{\mathrm{a}}$ \\ ${ }^{a}$ Dipt di Chimica, University of Bari, Via Orabona 4, I-70126 Bari, Italy \\ ${ }^{b}$ Istituto per i Processi Chimico-Fisici (IPCF), CNR - Via Orabona 4, I-70126 Bari, Italy \\ ${ }^{\mathrm{c}}$ CNR-IMIP, Sezione Territoriale di Bari, Via Orabona 4, I-70126 Bari, Italy \\ ${ }^{d}$ Dipt. Interateneo di Fisica, University of Bari, , Via Amendola 172, I-70126 Bari, Italy \\ ${ }^{\mathrm{e}}$ Dipt. of Fisiologia Generale e Ambientale, University of Bari, Via Orabona 4, I-70126 Bari, Italy \\ cotrone@,chimica.uniba.it
}

\begin{abstract}
Organic thin film transistor (OTFT) technology can be implemented to develop cost-effective and label-free bio-affinity sensor chips, having a field-effect transport directly coupled to a bio-sensing process, useful to high-throughput testing and pointof-care applications. Biological recognition elements such as antibodies or other proteins can be integrated in OTFT devices to confer specificity. In this study the use of lipid bilayers as support for biomolecules immobilization is investigated. Preliminary results in terms of electrical resistance and capacitance of the lipid bilayers are presented.
\end{abstract}

\section{INTRODUCTION}

To satisfy demands for fast and smart analytical systems a great deal of interest has been focused on the biosensors research area in recent years [1]. Several electrochemical (i.e, amperometric and potentiometric) and optical biosensors have been proposed for clinical, food or environmental applications $[2,3]$. However, miniaturization, integration on flexible substrate, low-cost fabrication techniques, signal amplification and label-free detection are still open issues.

In this respect, Organic electronics and OTFTs in particular appear as a new class of sensors able to overcome some of the commercial biosensor drawbacks $[4,5]$.

As far as volatile analytes are concerned, OTFT sensors have proven to offer the advantage of room temperature operation and the capability to deliver highly repeatable responses. Beside, they show quite good selectivity, though not specificity, by using properly chemically tailored organic semiconductor as active layers [6]. In addition to this, OTFTs are also able to offer the unique advantages of a multiparametric responses and a gate enhanced sensitivity.

Recently thin dielectric OTFTs have been demonstrated to operate at very low voltage, reaching even the sub-volt operating regime [7]. Their implementation in low power consumption devices has attracted the attention of the organic electronics community. Such low power transistors have also a great potential in sensing applications specifically those performed in a liquid environment. In fact, low voltage OTFTs have been recently demonstrated to deliver reliable responses even when operated in water for hundreds of measurement cycles. This opens new perspectives in the field of cheap, low-power and mass-produced aqueous biological sensors. Particularly, OTFT technology can be implemented to develop cost-effective and label-free DNA or protein sensor chips, with each single sensing element having the field-effect transport as much directly coupled to a biosensing process as possible [8].

The most important aspect in the design of biosensors is the choice of an immobilization strategy ensuring that the biological activity of the immobilized biomolecule is retained. Highly oriented bio-probes molecular layers on solid supports surfaces are required to attain selectivity as well as sensitivity and reproducibility for biosensing. Also in order to enhance sensitivity, the distance between the transducer surface and the immobilized biomolecules layer should be minimized [9, $10]$.

The biological sensing elements may be immobilized on the sensors surface via covalent binding, physical adsorption or entrapment in a polymer matrix. This can be achieved using a variety of substances including gels, lipid membranes, conducting polymers or self-assembled monolayers (SAMs) $[11,12]$. The use of cell-membrane mimics such as liposome and lipid bilayers has recently attracted the attention in biosensors development and several immobilization methods have been reported [13].

The intrinsically low bioactivity of supported membranes makes them interesting as an interface between the nonbiological material on the surface of a sensor and biologically active fluids. Lipid membranes also provide a natural environment for the immobilization of bioactive molecules such as enzymes or antibodies [14]. The natural environment for the active molecules offered by lipid membranes preserves their high sensitivity and selectivity by preventing denaturation, the low bioactivity of the supporting membrane reduces spurious signals, and the localization of the active molecules at a surface aids in signal transduction.

Lipid membranes may be deposited on a solid supports in several ways. In particular, the sensors surface can be modified to provide specific binding sites for lipid molecules 
thus allowing a stable immobilization of lipid membranes [15].

The goal of our work is to evaluate different approaches useful for biomolecules integration on OTFT devices. The coupling of the OTFT device and the biological recognition system is actuated by assembling supramolecular structures that integrate biomolecules deposited directly over the OTFT active layer. Bio-molecules are immobilized on the sensors surface using either conventional procedures (i.e, physisorption, chemisorption) and more innovative strategies based on the use of lipid layers hosting the selected biomolecules [18] (figure 1). In this respect, the investigation of the biomolecules or sBLM deposition and their electrical properties is one of the main steps to reach our aim. Particularly, in this paper impedance spectroscopy has been employed to evaluate electrical properties and the homogeneities of the lipid layers deposited on organic semiconductor. The advantage of this technique, is that the low biases preserve lipidic layers from destruction [17].

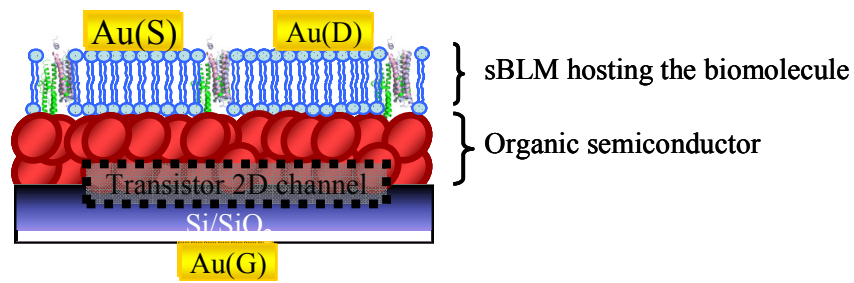

Fig. 1. Representation of a supported transmembrane protein OTFT.

\section{EXPERIMENTAL SECTION}

For impedance measurements the samples consist of a multilayer structure formed by an $\mathrm{Si} / \mathrm{SiO}_{2}$ substrate covered with a gold film 100nm thick on which is deposited an organic semiconductor (Sigma Chemical Co., St. Louis, MO) $\alpha, \omega$-dihexylsexithiophene $(\mathrm{DH} \alpha 6 \mathrm{~T})$. The gold and the organic semiconductor films are obtained by thermal evaporation and lipid vescicles suspension are dried on them under a nitrogen stream. To make the vescicles suspension, 10 $\mathrm{mg}$ of soy bean lecithin (EPIKURON 200 from Cargill) and $10 \mu \mathrm{g}$ of a fluorofore labeled dihexadecanoyl-phosphatidylethanolamine (Texas red DHPE from Invitrogen) were first dissolved in chloroform and dehydrated under vacuum). These molecules fluoresce at about $615 \mathrm{~nm}$ and the peak of their absorption spectrum is at $589 \mathrm{~nm}$. The dried mixtures were rehydrated in $1 \mathrm{~mL}$ of distilled water and sonicated on ice for $30 \mathrm{~min}$. The solution was then extruded several times through a polycarbonate filter with $50 \mathrm{~nm}$ diameter pores to produce vesicles of uniform size. The resultant small unilamellar vesicles (SUVs) were sized by dynamic light scattering using a ZetasizerNano (Malvern Instruments) and were found to be uniform with an average diameter of $82 \mathrm{~nm}$. Two class of experiments were performed: one with lipid vescicles suspension deposited directly on the gold film and the second on the organic semiconductor one.

Fluorescence images of lipid layers deposited on the organic semiconductor were acquired using an imaging setup running Metafluor software (Molecular Devices, MDS
Analytical Technologies, Toronto, Canada). Samples mounted on microscope slides were placed on the stage of a Nikon TE2000-U (Nikon Instruments Europe B.V., Bodhoevedorp, The Netherlands) and illuminated through a 4x Plan objective (Nikon Instruments) by monochromatic light. The emitted fluorescence was passed through a dichroic mirror and filtered through the appropriate emission filters (Omega Optical, Brattleboro, VT). Fluorescence images were captured by a cooled charge-coupled device camera (CoolSNAP HQ, Photometrics, Tucson, AZ) and converted to pseudocolored images by the Metafluor software. Impedance spectroscopy was performed by means of a HP 4194A Impedance-phase analyzer. With this instrument either the real and imaginary components of impedance can be directly obtained, or, equivalently, they can be output in terms of parallel conductance and capacitance of the sample. The last measurement option was chosen for the lipid layers/organic semiconductor systems and spectra were obtained in the frequency range $100 \mathrm{~Hz} \div 10 \mathrm{MHz}$ by applying small amplitude signal of $50 \mathrm{mV}$. The experimental behaviour was then analyzed by means of equivalent circuits representing the contribute of the various elements of the lipid layers/organic semiconductor systems. It must be underlined that best fit was achieved when substituting some ideal components with constant phase elements (CPE), which account for nonlinearities and frequency dependence of the elements by expressing the electrical impedance in terms of a simple power law:

$$
Z_{C P E}=K \omega^{-\alpha}
$$

$\omega$ being the angular frequency. If the frequency exponent is $\alpha=1$ then $\mathrm{K}=1 / \mathrm{C}$ and $Z_{C P E}$ represents an ideal capacitance. On the other hand, if $\alpha=0$ then $\mathrm{K}=\mathrm{R}$ and $Z_{C P E}$ stands for an ideal resistance [18].

All electrochemical measurements were carried out in aqueous $\mathrm{LiClO}_{4}$ solution $0.005 \mathrm{M}$.

\section{RESULTS AND DISCUSSION}

Capacitance and conductance measurements were performed on two different systems: gold/lipid layers and gold/organic semiconductor/lipid layers. In figures $2 \mathrm{~A}$ and $2 \mathrm{~B}$ the spectra of capacitance and conductance vs. the logarithm of frequency of the gold/lipid layers and gold/organic semiconductor/lipid layers systems are reported, respectively.

The comparison of the capacitance and conductance curves shown in Fig. 2, evidences that the system formed by semiconductor/lipid layers (Fig. 2B) shows trends similar to that of gold/lipid layers system (Fig. 2A). In both spectra the capacitance decreases with the frequency while the conductance increases. Furthermore, lipid layers and semiconductor/lipid layers show a capacitance of $100 \mathrm{nF}$ and $43 \mathrm{nF}$ while a conductance of $33 \mu \mathrm{S}$ and $14 \mu \mathrm{S}$, respectively indicating that the presence of semiconductor doesn't significantly affect the total capacitance and conductance.

As observed in recent papers, the contribution to capacitance and conductance at the lowest frequencies has been attributed to the lipid layer [19], whereas the electrolyte or the organic semiconductor is expected to influence the 
response at the highest frequencies. For the best of our knowledge, no mention has been made till now of the frequency response of the lipid layers/organic semiconductor system.
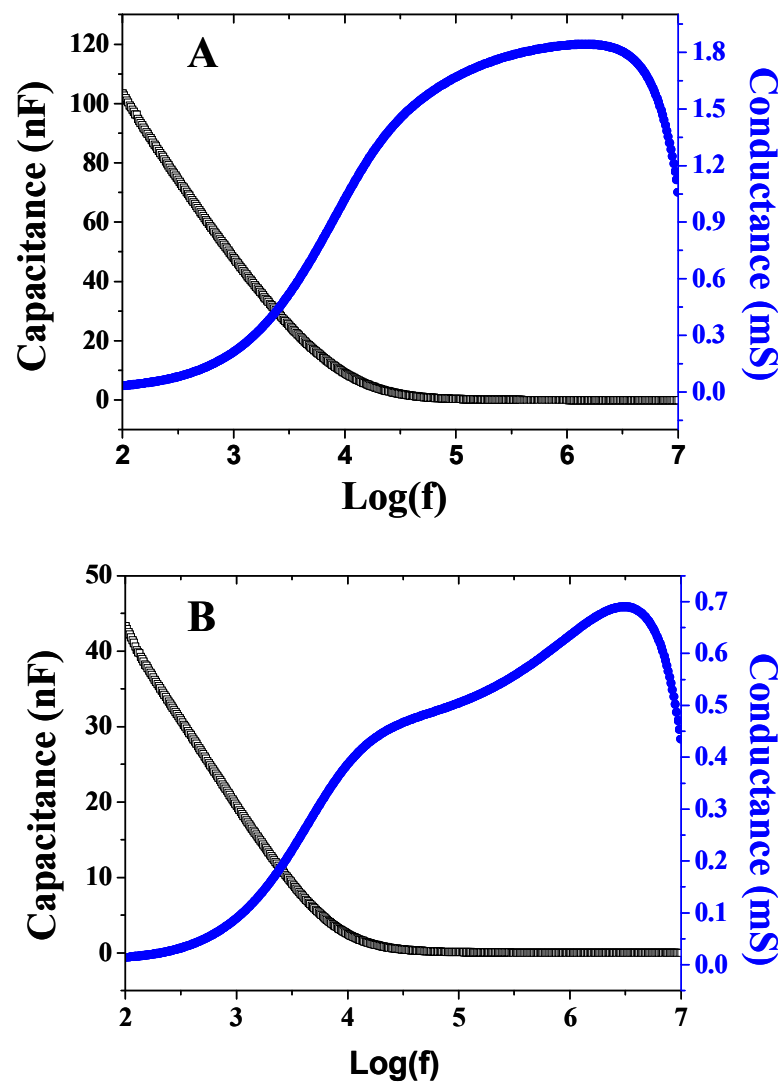

Fig. 2. Capacitance and conductance spectra of A) gold/lipid layers; B) gold/organic semiconductor/lipid layers.

Generally, the electrical properties of the gold/lipid layers and gold/organic semiconductor/lipid layers systems are analyzed by means of Nyquist plots, where experimental impedance $(Z)$ spectra are represented in terms of imaginary $\left(-Z_{\text {im }}\right)$ vs real $\left(Z_{\text {re }}\right)$ part of the impedance. The data analysis has been made by using a software packaging (EIS Spectrum analyser) allowing to fit $\left(-Z_{\text {im }}\right)$ vs real $\left(Z_{\text {re }}\right)$ with those resulting by modelling the structure under test by means of suitable equivalent circuit. In Fig. 3 it has been represented the experimental Nyquist plot (triangles) and corresponding best fit (red line) obtained for one of the examined lipid layers /organic semiconductor structure. In the same Figure, the equivalent circuit allowing the best fit has been reported. Since the system under investigation is constituted by superimposed layers, the equivalent circuit has been considered as resulting from three different contributions: the first one, is represented by the parallel between the capacitance $\left(\mathrm{C}_{\text {S.O. }}\right)$ with the resistance $\left(\mathrm{R}_{\text {S.O. }}\right)$ of organic semiconductor. The second one corresponding to the lipid layers has been represented by a "constant phase element" $\left(\mathrm{CPE}_{\mathrm{L}}\right)$ in parallel with resistance $\left(\mathrm{R}_{\mathrm{L}}\right)$.

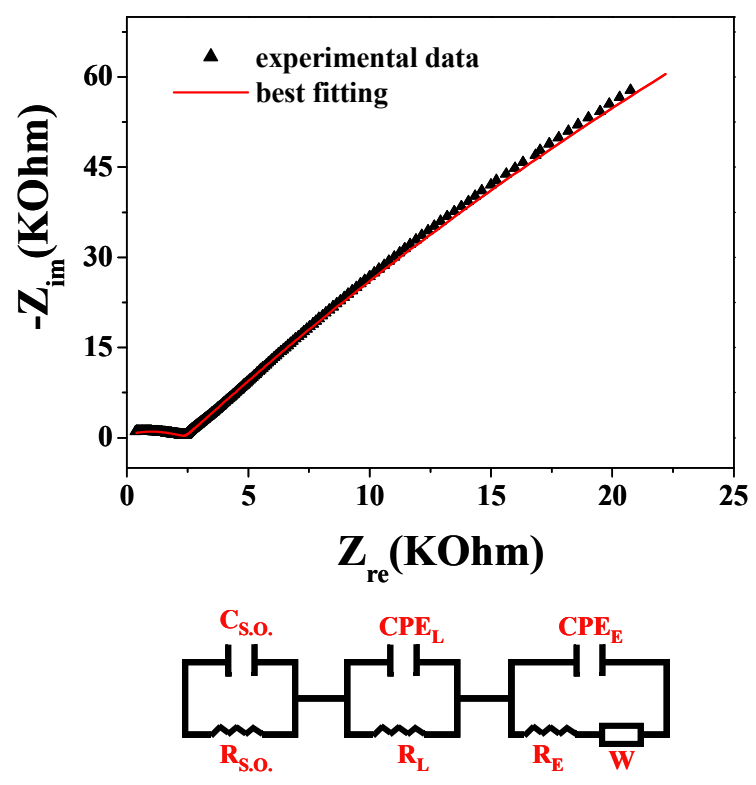

Fig. 3. Nyquits plot obtained from impedance spectra of gold/organic semiconductor/lipid layers system. Solid line represents the simulated curve according to the inserted circuit.

The choice of $\mathrm{CPE}_{\mathrm{L}}$ elements instead of pure capacitance element is justified because of the expected in-homogeneities of the lipid layers. Finally, the electrolyte contribution is represented by a "constant phase element" $\left(\mathrm{CPE}_{\mathrm{E}}\right)$ in parallel with resistance $\left(\mathrm{R}_{\mathrm{E}}\right)$ and a Warburg impedance $(\mathrm{W})$. The parameters obtained by the best fit evidenced and confirmed that the low frequency contribution (higher impedance part of Nyquist plot) is mainly due to lipid layer. Furthermore, the value of the fitting parameter $\alpha$ was found $\alpha=0.8$ for the $\mathrm{CPE}_{\mathrm{L}}$ thus indicating a significant in-homogeneity degree of the lipid film.

This result is confirmed also by fluorescence images obtained for lipid vescicles suspension deposited directly on the semiconductor layer.

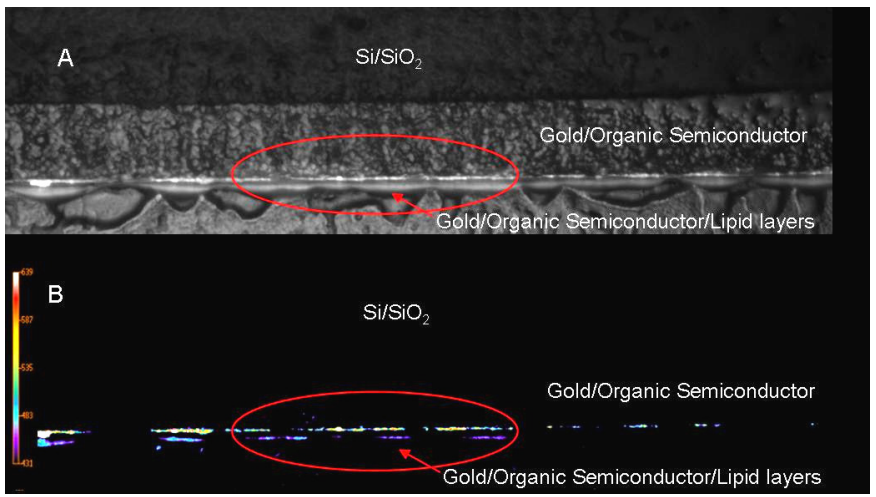

Fig. 4. Fluorescence (A) and pseudocolor (B) images of lipid layers deposited on the organic semiconductor. A section of the region considered in the impedance measurements is delimitated by red circle. 
The B panel of the Fig. 4. shows the fluorescence intensity as a colour code image, where blue means a low fluorescent signal while red is an high one. It is clearer from such a picture that besides seeing a quite non uniform fluorescence intensity there are regions that are not fluorescing at all confirming a not homogenous deposition of lipid layers.

\section{CONCLUSION}

In the present work lipid layers have been characterized by impedance spectroscopy. In particular capacitance and conductance measurements were performed on two different systems: gold/lipid layers and gold/organic semiconductor/lipid layers. Furthermore, information on the presence of lipid layer inhomogeneities are gathered from the electrical parameters determined by fitting the frequencydependent impedance of the equivalent circuits to the measured data. Preliminary results show that besides the inhomogeneity degree of the lipid film, confirmed by fluorescence images, lipid layers can be employed in OTFT device thanks to their electrical properties. However, the optimization of the lipid vescicles deposition on the organic semiconductor is a critical factor in the sensor development and future study will be addressed to improve this aspect.

\section{ACKNOWLEDGEMENTS}

\author{
"Electrolyte-Gated Organic Field-Effect Biosensors- \\ BioEGOFET" SEVENTH FRAMEWORK PROGRAMME- \\ THEME ICT-2009 and "Gas Sensors on Flexible Substrates \\ for Wireless Applications-FlexSMELL" MARIE CURIE \\ INTERNATIONAL TRAINING NETWORK - 7PQ-PEOPLE- \\ ITN-2008 supported all work described.
}

\section{REFERENCES}

[1] C. Bartic, G. Borghs, "Organic thin-film transistors as transducers for (bio)analytical applications," Anal Bioanal Chem, vol 384, pp. 354365 , September 2005.

[2] S. M. Borisov and O. S. Wolfbeis, "Optical biosensors," Chem. Rev., vol. 108, pp. 423-461, January 2008.

[3] D. Grieshaber, R. MacKenzie, J. Vörös and E. Reimhult, "Electrochemical Biosensors-Sensor Principles and Architectures," Sensors, vol. 8, pp.1400-1458, March 2008.

[4] N.A. Sokolov, M.R. Roberts and Z. Bao, "Fabrication of low cost electronic biosensors," Materials today, vol. 12, pp.12-20, September 2009.

[5] L. Torsi, G.M. Farinola, F. Marinelli, M.C. Tanese, O.H. Omar, L. Valli, F. Babudri, F. Palmisano, P.G. Zambonin and F. Naso, "A sensitivityenhanced field-effect chiral sensor," Nat Mater., vol 7, pp. 412 - 417 April 2008.

[6] L. Torsi, "Organic thin-film transistors as analytical and bioanalytical sensors," Anal Bioanal Chem, vol 384, pp. 309, November 2005.

[7] L. Torsi, G. Palazzo, A. Mallardi, M.D. Angione and S. Cotrone "Bioactive Supramolecular Architectures in Electronic Sensing Devices" in Functional Supramolecular Architectures, K. Sikora and K.E. Halnan, Eds, John Wiley \& Sons, Ltd, submitted

[8] F. Yan, S. M. Mok, J. Yu, H. L.W. Chan and M. Yang, "Label-free DNA sensor based on organic thin film transistors," Biosens. and Bioelectron., vol 24, pp. 1241-1245, January 2009.

[9] J. Turkova, "Oriented immobilization of biologically active proteins as a tool for revealing protein interactions and function," J. Chromatogr. B. Biomed. Sci. Appl., vol 722, pp. 11-31, February 1999.

[10] I. Vikholm, "Self-assembly of Antibody Fragments and Polymers onto Gold for Immunosensing," Sens. Actuators B, vol 106, pp. 311-316, July 2005.
[11] A. Subramanian, J. Irudayaraj and T. Ryan, "A mixed self-assembled monolayer-based surface plasmon immunosensor for detection of E. coli O157:H7," Biosens. Bioelectron., vol 21, pp. 998-1006, January 2006.

[12] B. Deore, Z. Chen and T. Nagaoka, "Potential-Induced Enantioselective Uptake of Amino Acid into Molecularly Imprinted Overoxidized Polypyrrole," Anal. Chem., vol 72, pp. 3989-3994, August 2000.

[13] H. Y. Lee, H. S. Jung, K. Fujikawa, J. W. Park, J. M. Kim, T. Yukimasa, H.Sugihara and T. Kawai, "New antibody immobilization method via functional liposome layer for specific protein assays," Biosens. Bioelectron., vol 21, pp. 833-838, November 2005.

[14] M. Stelzle, G. Weissmueller, E. Sackmann, "On the application of supported bilayers as receptive layers for biosensors with electrical detection," J. Phys. Chem., vol 97, pp. 2974-2981, March 1993.

[15] M. Indrieri, M. Suardi, A. Podest, E. Ranucci, P. Ferruti and P. Milani, "Quantitative investigation by atomic force microscopy of supported phospholipid layers and nanostructures on cholesterol-functionalized glass surfaces," Langmuir, vol 24, pp. 7830-7841, July 2008.

[16] L. Torsi, M. D. Angione, S. Cotrone, "Organic electronic biological Sensing," SPIE Newsroom, DOI 10.1117/2.1200909.1727.

[17] V. I. Passechnik, T. Hianik, S. A. Ivanov, B. Sivak, "Specific capacitance of metal supported lipid membranes," Electroanalysis, vol 10, pp. 295-302, April 1998.

[18] H. Hillebrandt, G. Wiegand, M. Tanaka, and E. Sackmann, "High electric resistance polymer/lipid composite films on indium-tin-oxide electrodes," Langmuir, vol 15, pp. 8451-8459, September 1999.

[19] Y. Shao, Y. Jin, J. Wang, L. Wang, F. Zhaoand S. Dong, "Conducting polymer polypyrrole supported bilayer lipid membranes," Biosens. Bioelectron., vol 20, pp. 1373-1379, June 2004. 